\title{
Obtención de nanoemulsiones utilizando saponinas de quillay como sustituto de un surfactante sintético*
}

\section{Obtaining nanoemulsions using quillay saponins as a substitute for a syntethic surfactant}

\author{
Riquelme, N. ${ }^{* *, * * *}$ y Arancibia, C. ${ }^{* *, 1}$
}

\begin{abstract}
The objective of this work was to study synthetic surfactant substitution for natural origin one and to evaluate its effect on physical and oxidative stability of healthy nanoemulsions containing avocado oil. Healthy nanoemulsions were prepared using avocado oil as a lipid phase (5\% wt/wt) and an aqueous phase consisting of water (89\% wt/wt) and different surfactants (6\% wt/wt): soy lecithin, Tween 80 and quillaja saponins, replacing Tween 80 by quillaja saponins from $0 \%$ to $100 \%$. Physical characteristics (particle size, polydispersity index and zeta potential), and physical and oxidative stability of nanoemulsions were determined. Results showed that Tween 80 substitution by saponins affected significantly $(p<0.05)$ physical characteristics of nanoemulsions, since saponins were less efficient on particle size reduction; however, it was obtained a slight effect on polydispersity index (0.17-0.22) and zeta potential $(-38.6--49.9 \mathrm{mV})$. In conclusion, quillaja saponins can be used as a substitute for a synthetic surfactant for development of healthy nanoemulsions with good physical and oxidative stability, however this natural surfactant is less efficient in obtaining particle sizes in nanometric scale $(<100 \mathrm{~nm})$.
\end{abstract}

KEYWORDS: nanoemulsions, quillaja saponaria, physical stability, oxidative stability, avocado oil.

RESUMEN: El objetivo de este trabajo fue estudiar la sustitución de un surfactante sintético (Tween 80 ) por uno de origen natural (saponinas de quillay) y evaluar su efecto sobre la estabilidad fisica y oxidativa de nanoemulsiones saludables con aceite de palta (aguacate). Se prepararon nanoemulsiones con aceite de palta como fase lipídica saludable $(5 \% p / p)$ y una fase acuosa constituida por agua ( $89 \% \mathrm{p} / \mathrm{p})$ y distintos surfactantes $(6 \% \mathrm{p} / \mathrm{p})$ : lecitina de soya, Tween 80 y saponinas de quillay, sustituyendo el Tween 80 por saponinas de quillay de $0 \%$ a $100 \%$. Se determinaron las propiedades físicas (tamaño de partícula, índice de polidispersidad y potencial zeta) y la estabilidad fisica y oxidativa de las nanoemulsiones. Los resultados mostraron que la sustitución con saponinas afectó significativamente $(p<0.05)$ las características físicas de las

Recibido: 4 de noviembre de 2018.

Aceptado: 18 de diciembre de 2018.

* Los autores agradecen el apoyo económico de Conicyt por el Proyecto PAI No 79160091 de la Dra. Carla Arancibia y por la beca de doctorado nacional de la Mg. Natalia Riquelme. A las empresas Blumos S. A. y Naturex S. A. por proporcionar muestras gratuitas de lecitina de soya y saponinas de quillay, respectivamente.

** Universidad de Santiago de Chile, Santiago, Chile. Departamento de Ciencia y Tecnología de los Alimentos, Facultad Tecnológica.

*** Universidad de Chile, Santiago, Chile. Instituto Nutrición y Tecnología de Alimentos.

${ }^{1}$ Autora de correspondencia: carla.arancibia@usach.cl 
Mundo Nano | ARTículos | www.mundonano.unam.mx

12(23), 1e-12e, julio-diciembre 2019 | http:// dx.doi.org/10.22201/ceiich.24485691e.2019.23.67654

Riquelme N. y Arancibia C.

nanoemulsiones, las saponinas presentaron una menor efectividad en la reducción del tamaño de partícula; sin embargo, mostraron un leve efecto sobre el índice de polidispersión (0.17-0.22) y potencial zeta (-38.6 - -49.9 mV). En conclusión, las saponinas de quillay pueden ser utilizadas como sustituto de un surfactante sintético para el desarrollo de nanoemulsiones saludables con buena estabilidad fisica y oxidativa; no obstante, este surfactante natural es menos eficiente en la obtención de tamaños de partícula en escala nanométrica $(<100 \mathrm{~nm})$.

PALABRAS CLAVE: nanopartículas, nanomateriales, individuos, materia, ontología.

\section{Introducción}

El desarrollo de nanoemulsiones como sistemas de liberación de compuestos bioactivos lipídicos se ha investigado debido a su potencial uso en la industria de alimentos (Bai et al., 2016), pues presentan diversas ventajas como: buena dispersibilidad en agua, claridad óptica, mejor estabilidad física en términos de separación gravitacional y mayor biodisponibilidad lipídica (Salvia-Trujillo et al., 2017) debido a su reducido tamaño promedio de partícula (<200 nm) (Gupta et al., 2016; Montes de Oca-Ávalos et al., 2017). Desde el punto de vista termodinámico, las nanoemulsiones tienden a desestabilizarse durante el almacenamiento, siendo necesario un agente surfactante para mejorar su estabilidad física (McClements y Jafari, 2017), por su rol en la reducción de la tensión interfacial que facilita la formación de gotas pequeñas durante el proceso de homogeneización (Gupta et al., 2016) y en la generación de interacciones repulsivas (como estéricas o electrostáticas) entre las gotas de aceite que evitan su agregación (Bai et al., 2016). De las cualidades que debe tener un surfactante para estabilizar emulsiones, se encuentran: a) rápida absorción en la superficie de las gotas de aceite durante el proceso de homogeneización con el fin de reducir la tensión interfacial y facilitar la disrupción de las gotas; b) formación de una capa protectora estable alrededor de las gotas de aceite para evitar su agregación durante la elaboración, el transporte y el almacenamiento; c) fácil uso, amplia disponibilidad y calidad confiable, y, d) ser económicamente viable (Chung et al., 2017). Además, en la formulación y desarrollo de alimentos la selección del tipo de surfactante se debe también considerar el perfil de sabores que puede impartir en el producto junto con su compatibilidad con los otros ingredientes e incluso el estatus legal (Luo et al., 2017).

Entre los surfactantes sintéticos más utilizados en la elaboración de emulsiones se encuentran los ésteres de polioxietilen sorbitano, conocidos como Tween debido a sus buenas propiedades emulsionantes y su bajo costo (Raikos et al., 2016). Este surfactante no iónico tiene un HLB (hydrophilic-lipophilic balance) igual a 15, lo cual permite, efectivamente, estabilizar nanoemulsiones del tipo O/W (aceite en agua) y posee una baja toxicidad en comparación con otros surfactantes sintéticos (McClements, 2015). De este modo, en el último tiempo, la industria alimentaria está buscando remplazar estos surfactantes sintéticos por otros naturales o de origen botánico debido 
a una mayor demanda de los consumidores por alimentos más saludables, naturales y "amigables" con el medio ambiente (McClements et al., 2017). Por ello, se han investigado nuevas fuentes de surfactantes naturales, tales como: polisacáridos, proteínas, fosfolípidos y pequeñas moléculas surfactantes como las saponinas (McClements, 2015; Luo et al., 2017).

Las saponinas han despertado gran interés en la industria alimentaria por sus buenas propiedades emulsionantes y espumantes (Zhang et al., 2015). Son moléculas relativamente pequeñas formadas por una aglicona (hidrofóbica) y una o más cadenas de azúcar (hidrofílicas) que les otorga su naturaleza anfifílica y un gran número de propiedades funcionales-tecnológicas. El uso de las saponinas como surfactante tiene diversas ventajas: son naturales, biodegradables, sustentables y presentan baja toxicidad (Uzoigwe et al., 2015; McClements et al., 2017) al presentar un IDA (ingesta diaria admisible) igual a $5 \mathrm{mg} / \mathrm{Kg}$ de peso corporal (WHO, 2006). Diversos estudios han investigado las propiedades interfaciales de las saponinas de quillay (extraída de la corteza del árbol Quillaja saponaria Molina, normalmente encontrado en Chile), tanto su utilización en emulsiones (Yang et al., 2013; Bai et al., 2016) y espumas (Böttcher y Drusch, 2016), y/o en modelos de alimentos como la crema para café (Chung et al., 2017). No obstante, existe poca información de su utilización en la elaboración de nanoemulsiones alimentarias (Zhu et al., 2019). Por ello, el objetivo de este trabajo de investigación fue estudiar la sustitución de un surfactante sintético (Tween 80) por uno de origen natural (saponinas de quillay) en la elaboración de nanoemulsiones con aceite de palta evaluando su efecto sobre la estabilidad física y oxidativa durante su almacenamiento.

\section{Materiales y métodos}

\section{Materiales}

Los ingredientes utilizados en la elaboración de las nanoemulsiones del tipo aceite en agua (O/W) fueron: (1) fase acuosa: agua purificada obtenida mediante un sistema de ósmosis inversa (Vigaflow S.A., Chile), (2) fase lipídica: aceite de palta (Casta de Peteroa - Terramater S.A., Chile), y, (3) agentes surfactantes: lecitina de soya (Metarin P - Blumos S.A., Chile), Tween 80 (Sigma-Aldrich S.A., Estados Unidos) y el extracto con saponinas de quillay (Quillaja saponaria Molina) (Sapnov L - Naturex S.A., Chile). Para el estudio de la estabilidad física de las nanoemulsiones frente al $\mathrm{pH}$ se utilizó ácido clorhídrico (J.T. Baker Inc., Estados Unidos) e hidróxido de sodio (Winkler Ltda., Chile), y en los ensayos de oxidación lipídica se utilizaron ácido tricloroacético, ácido tiobarbitúrico y malonaldehído (Merck S.A., Chile).

\section{Preparación de las nanoemulsiones}

Para la preparación de las nanoemulsiones, en primer lugar, se realizó una pre-emulsión con el fin de dispersar la fase lipídica ( $5 \%$ p/p) en la fase acuo- 
sa $(95 \% \mathrm{p} / \mathrm{p})$ que consistió en un $89 \% \mathrm{p} / \mathrm{p}$ de agua purificada y un $6 \% \mathrm{p} / \mathrm{p}$ de una mezcla de surfactantes: $5 \% \mathrm{p} / \mathrm{p}$ de lecitina de soya y $1 \% \mathrm{p} / \mathrm{p}$ de la proporción Tween 80/saponinas de quillay, donde la concentración de Tween 80 se sustituyó por saponinas de quillay a distintos porcentajes: $0,25,50,75 \mathrm{y}$ $100 \%$. La formación de la pre-emulsión se llevó a cabo con un dispersor de alta velocidad (Ultraturrax, IKA T25, Alemania) a 10,000 rpm durante 5 min, en un baño de hielo para evitar el sobrecalentamiento de la muestra. En una segunda etapa, la pre-emulsión se sometió a un tratamiento por ultrasonido (VCX500, Sonics, Estados Unidos) utilizando: $20 \mathrm{KHz}$ de frecuencia, un 90\% de amplitud de onda y 20 min de homogeneización. Una vez obtenidas las nanoemulsiones se almacenaron en frascos de vidrio a $4{ }^{\circ} \mathrm{C}$ para su posterior caracterización.

\section{Caracterización de las nanoemulsiones}

Tamaño de partícula e índice de polidispersidad

El tamaño de partícula (PS) y el índice de polidispersidad (PdI) de las diferentes nanoemulsiones se determinaron mediante dispersión de luz dinámica (DLS) utilizando un Zetasizer (NanoS90, Malvern Instruments, Reino Unido). Para realizar las mediciones, las nanoemulsiones se diluyeron al $6 \% \mathrm{v} / \mathrm{v}$ en agua mili-Q con el fin de obtener una solución casi transparente. Se utilizó un índice de refracción de la fase dispersa lipídica igual a 1.47 y una temperatura de $25^{\circ} \mathrm{C}$. Los valores informados corresponden a un promedio de 10 mediciones por duplicado y cada muestra se midió por triplicado.

\section{Potencial Zeta}

La carga eléctrica (potencial zeta) de las nanoemulsiones se caracterizó mediante un Zetasizer (Nano-ZS, Malvern Instruments, Reino Unido). Las nanoemulsiones se diluyeron en agua mili-Q en un $20 \%$ v/v y se depositaron en celdas capilares equipadas con dos electrodos (celdas capilares desechables, Malvern Instruments, Reino Unido). Se utilizó un índice de refracción de la fase dispersa lipídica igual a 1.47 y una temperatura igual a $25^{\circ} \mathrm{C}$. Los valores de ZPot se recolectaron con base en 30 lecturas continuas donde cada muestra se midió por triplicado.

\section{Estabilidad física de las nanoemulsiones}

\section{Estabilidad fisica durante el almacenamiento}

Se estudió la estabilidad física de las nanoemulsiones saludables durante su almacenamiento a 5 y $25^{\circ} \mathrm{C}$. Para ello, $8 \mathrm{ml}$ de cada muestra se colocaron en un tubo de vidrio de $12 \mathrm{~cm}$ de altura, y se almacenaron por 30 días midiendo la formación de crema (índice de cremado) en función del tiempo de almacenamiento $(0,5,10,15,20$ y 30 días). El índice de cremado se calculó usando la siguiente ecuación: 


$$
\text { Índice de cremado }(\%)=\frac{A C}{A T} \times 100
$$

en donde, $A C$ es la altura de la capa de crema $(\mathrm{mm})$ y $A T$ es la altura total de la nanoemulsión ( $\mathrm{mm}$ ).

\section{Efecto del $p H$}

Se analizó el efecto del pH sobre el tamaño de partícula (PS) de las nanoemulsiones y en su estabilidad, luego de un proceso de centrifugación a 4,539 rpm por 15 min (Universal 32R, Hettich, Reino Unido), de acuerdo con la metodología de Bortnowska et al. (2014). El pH de las diferentes muestras se ajustó con un medidor de pH (HI 111, Hanna Instruments, Rumania) utilizando $\mathrm{HCl}$ o $\mathrm{NaOH} 1 \mathrm{M}$ para obtener un $\mathrm{pH}$ final en un rango entre 3 y 8.

\section{Estabilidad oxidativa de las nanoemulsiones}

La estabilidad oxidativa se determinó a través de la cuantificación de las sustancias reactivas al ácido tiobarbitúrico (TBARs) siguiendo la metodología de Arancibia et al. (2017). Para cuantificar los productos de la oxidación lipídica se mezclaron $0.3 \mathrm{ml}$ de cada nanoemulsión con $2 \mathrm{ml}$ de una solución de ácido tiobarbitúrico ( $15 \% \mathrm{p} / \mathrm{v}$ de ácido tricloroacético y $0.375 \% \mathrm{p} / \mathrm{v}$ de ácido tiobarbitúrico en ácido clorhídrico $0.25 \mathrm{M}$ ) y $1 \mathrm{ml}$ de agua mili-Q. La mezcla se calentó a $90^{\circ} \mathrm{C}$ por 15 min en un baño termoregulado (B-100, Buchi, Suiza), luego se enfrió y se centrifugó a 3,400 rpm durante 15 min (MiniSpin Plus, Eppendorf, Alemania). Finalmente, se midió la absorbancia de las muestras a 532 y $580 \mathrm{~nm}$ en un lector de microplacas (Multiskan Go, Thermo Scientific, Estados Unidos), donde la absorbancia final correspondió a la diferencia de la absorbancia a 532-580 $\mathrm{nm}$. Las concentraciones de TBARs se calcularon a partir de una curva patrón preparada con una solución de malonaldehído $12 \mathrm{mM}$.

\section{Análisis estadísticos}

Todos los experimentos se realizaron por triplicado y los resultados fueron informados mediante su promedio y desviación estándar. El análisis estadístico se realizó mediante ANOVA (análisis de varianza) y el post-test de Tukey con un nivel de significancia del 95\% utilizando el software XLSTAT@ 2018.2 50198 (Addinsoft, Francia).

\section{Resultados y discusión}

\section{Caracterización de las nanoemulsiones}

El tamaño de partícula (PS) de las diferentes nanoemulsiones se muestra en la figura $1 \mathrm{~A}$, donde se observa que al aumentar el porcentaje de saponinas de quillay (SQ) los valores de PS se incrementan significativamente $(\mathrm{p}<0.05)$ desde 90 a $206 \mathrm{~nm}$ para 0 y 100\% SQ, respectivamente. No obstante, todas 
FIGURA 1. Valores promedio y diferencias significativas para A) tamaño de partícula; B) índice de polidispersidad, y, C) potencial zeta de las nanoemulsiones elaboradas con diferentes porcentajes de saponinas de quillay (SQ) $(0-100 \% \mathrm{p} / \mathrm{p})$.
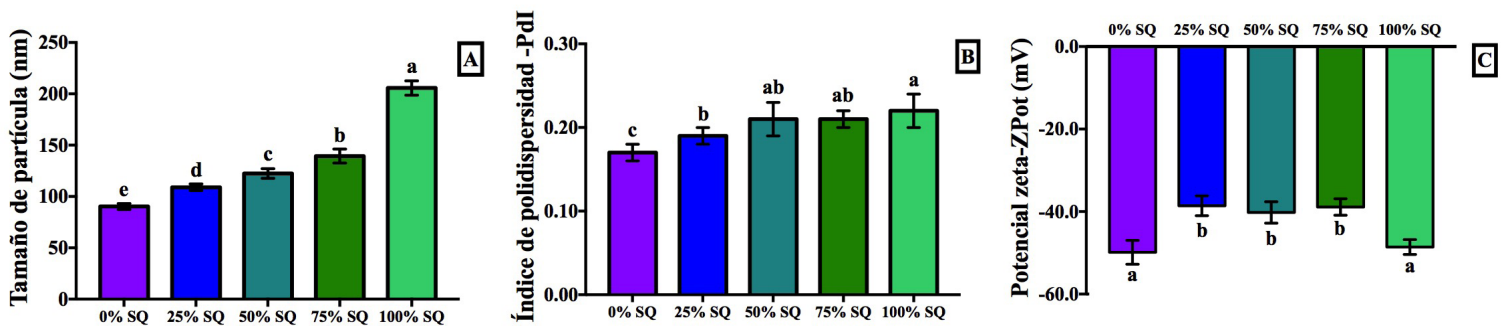

Fuente: Elaboración de los autores.

las nanoemulsiones elaboradas con la mezcla Tween 80 - SQ presentaron tamaños de partícula $<150 \mathrm{~nm}$. Estos resultados están de acuerdo con los obtenidos por Yang et al. (2013), quienes reportaron que la mezcla de surfactantes Tween 80 y saponinas de quillay es efectiva para generar pequeñas gotas dispersas en sistemas con triglicéridos de cadena media (MCT), aunque el Tween 80 por sí mismo resultó ser más eficiente que las saponinas de quillay en la reducción del PS. Esto se puede deber a una diferencia en la velocidad de adsorción en la interfase aceite-agua y de la capacidad de reducir la tensión interfacial que tiene cada surfactante (Luo et al., 2017), lo que afecta la reducción del PS durante el proceso de homogeneización. En el caso del índice de polidispersidad (PdI), se observó una variación leve al aumentar el porcentaje de saponinas de quillay, observándose diferencias significativas $(p<0.05)$ solo entre los niveles 0.25 y $100 \%$ SQ (figura 1B). Además, cabe destacar que todos los valores de PdI estuvieron en el rango de $0.17 \mathrm{a}$ 0.22 , representando una distribución monomodal de los tamaños de partícula, que podría indicar una buena estabilidad física de las nanoemulsiones durante su almacenamiento (Silva et al., 2015).

En la figura $1 \mathrm{C}$ se observan los valores de potencial zeta (ZPot) de las distintas nanoemulsiones, los cuales muestran una carga eléctrica negativa $(\approx-40 \mathrm{mV}$ ) debido principalmente a la carga eléctrica de dos de los surfactantes utilizados en su elaboración: lecitina de soya y saponinas de quillay (Öztürk y McClements, 2016), puesto que Tween 80 es un surfactante no iónico que tiene carga eléctrica neutra (Arancibia et al., 2017). La lecitina de soya tiene carga negativa debido a la presencia de fosfolípidos aniónicos en su cabeza polar que está constituida por un grupo fosfato y un aminoalcohol (McClements y Gumus, 2016), como fosfatidilcolina, fosfatidiletanolamina y fosfatidilinositol en concentraciones iguales a 24,17 y $14 \%$, respectivamente (según lo reportado por el proveedor). Mientras que las saponinas de quillay presentan una carga negativa por el grupo ácido carboxílico presente en su estructura (Zhu et al., 2019). Por otro lado, la utilización de la mezcla del Tween 80-SQ disminuyó significativamente $(\mathrm{p}<0.05)$ la electronegati- 
vidad de las nanoemulsiones en comparación con los controles 0\% SQ (solo Tween 80 ) y $100 \%$ SQ (solo saponinas de quillay) (figura 1C), independientemente del porcentaje de saponinas de quillay utilizado, lo que se puede atribuir a la forma en que se posicionan los distintos surfactantes en la interfase aceite-agua ya que probablemente se intercalan en la interfase formando un sistema miscible con distribución aleatoria, lo cual puede disminuir la electronegatividad de la partícula coloidal (McClements y Jafari, 2017).

\section{Estabilidad física de las nanoemulsiones}

Todas las nanoemulsiones se mantuvieron estables durante su almacenamiento a 5 y $25^{\circ} \mathrm{C}$ ya que no se observó formación de cremado (figura 2). Este comportamiento se puede atribuir a los resultados obtenidos de los parámetros físicos de PS, PdI y ZPot (figura 1). En el caso del tamaño de partícula, si bien se observaron diferencias significativas $(p<0.05)$ entre los distintos porcentajes de saponinas de quillay (figura 1A), la obtención de PS $<200 \mathrm{~nm}$ provocó una mayor estabilidad física durante el almacenamiento, pues en estos valores de PS predominan las fuerzas brownianas y disminuyen las fuerzas atractivas entre las gotas lo que evita su agregación y, por lo tanto, mejora su estabilidad física (McClements, 2015). El índice de polidispersidad también tiene un papel fundamental en la estabilidad física de las nanoemulsiones donde valores de $\mathrm{PdI}<0.2$, como los obtenidos en las nanoemulsiones con distintos porcentajes de saponinas de quillay (figura 1B), indican una alta estabilidad física debido a la homogeneidad en la distribución de los tamaños de partícula. En adición, considerando que los valores absolutos de ZPot de todas las nanoemulsiones fueron $>30 \mathrm{mV}$ (figura $1 C$ ) se puede decir que existió una mayor repulsión eléctrica de las gotas de aceite, lo que ayudó a mantener su estabilidad física (McClements y Jafari, 2017). Por lo tanto, las saponinas de quillay fueron tan efectivas como el Tween 80 en mantener la estabilidad física de las nanoemulsiones durante su almacenamiento.

FIGURA 2. Fotografias de la estabilidad física de las nanoemulsiones con diferentes porcentajes de saponinas de quillay (SQ) $(0-100 \% \mathrm{p} / \mathrm{p})$ durante su almacenamiento a $25^{\circ} \mathrm{C}$, para el día 0 (A) y día 30 (B).
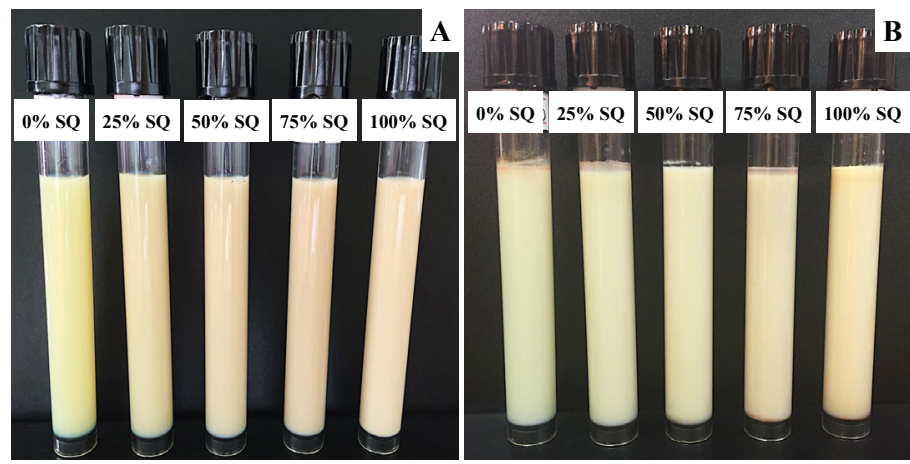

Fuente: Fotografias tomadas por los autores. 
Cabe destacar que se observó (figura 2) un cambio en el color de las distintas nanoemulsiones al aumentar la concentración del extracto de saponinas (0 a 100\% SQ), desde un color amarillo-pálido a un amarillo-marrón, lo que se pudo deber al color intrínseco del extracto con saponinas de quillay (marrón oscuro) (Chung et al., 2017). Sin embargo, estas diferencias disminuyeron durante el almacenamiento sugiriendo que la pigmentación del extracto con saponinas de quillay no fue estable durante el almacenamiento.

Respecto al efecto del $\mathrm{pH}$ sobre el tamaño de partícula se puede decir que no se observaron cambios en los valores de tamaño de partícula bajo los distintos tratamientos de $\mathrm{pH}$ (figura 3), manteniéndose las diferencias observadas en el PS debido a la composición de las nanoemulsiones (diferente porcentaje de Tween 80 y saponinas de quillay).

Además, se observó que las nanoemulsiones elaboradas con distintos porcentajes de saponinas de quillay (0-100\% SQ) se mantuvieron visiblemente estables luego de ser sometidas a un proceso acelerado de desestabilización por centrifugación a todos los $\mathrm{pH}$ estudiados (figura 4). La buena estabilidad que presentaron las nanoemulsiones con saponinas de quillay (25-100\% SQ) se pudo deber a que las saponinas de quillay presentan una alta estabilidad en un amplio rango de $\mathrm{pH}$ de 3 a 8 (Öztürk et al., 2014), ya que a valores de $\mathrm{pH}$ más altos las saponinas se encuentran cargadas más negativamente, lo cual previene la agregación de las gotas de aceite y con ello la desestabilización de las nanoemulsiones (McClements y Gumus, 2016). La nanoemulsión control ( $0 \%$ SQ) elaborada sin saponinas de quillay también presentó una buena estabilidad física a los distintos $\mathrm{pH}$, lo cual puede deberse a la buena capacidad emulsificante del Tween 80 que previene la agregación de las gotas de aceite bajo estas condiciones de $\mathrm{pH}$ (Uluata et al., 2015). Cabe destacar, que en la figura 4 se observa un precipitado en las distintas nanoemulsiones que corresponde a sedimentos propios del extracto de quillay, el cual aumentó a medida que se incrementaba la concentración de saponinas (0-100\% de saponinas de quillay - SQ).

FIGURA 3. Efecto del pH en el tamaño de partícula (PS) de las nanoemulsiones con diferente porcentaje de saponinas de quillay (SQ) (0-100\% p/p). Los valores se expresan como promedio y desvío estándar.

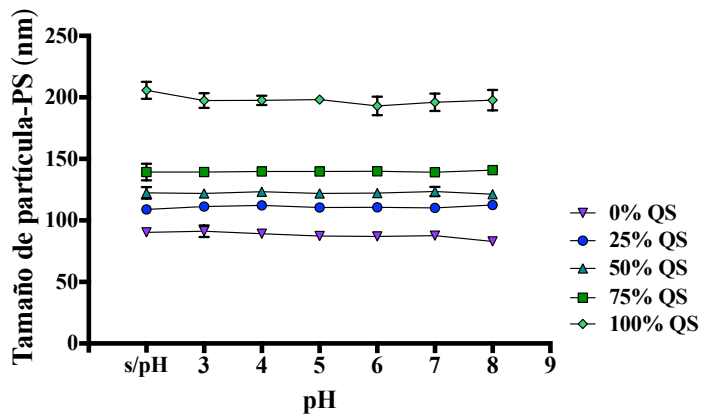

Fuente: Elaboración de los autores. 
FIGURA 4. Fotografias de las nanoemulsiones con diferente porcentaje de saponinas de quillay (SQ) $(0-100 \% \mathrm{p} / \mathrm{p})$ y diferentes tratamientos de $\mathrm{pH}(3-8)$ después de un proceso de centrifugación a 4,539 rpm por $15 \mathrm{~min}$. El precipitado de color marrón corresponde a la sedimentación del extracto de saponinas de quillay.

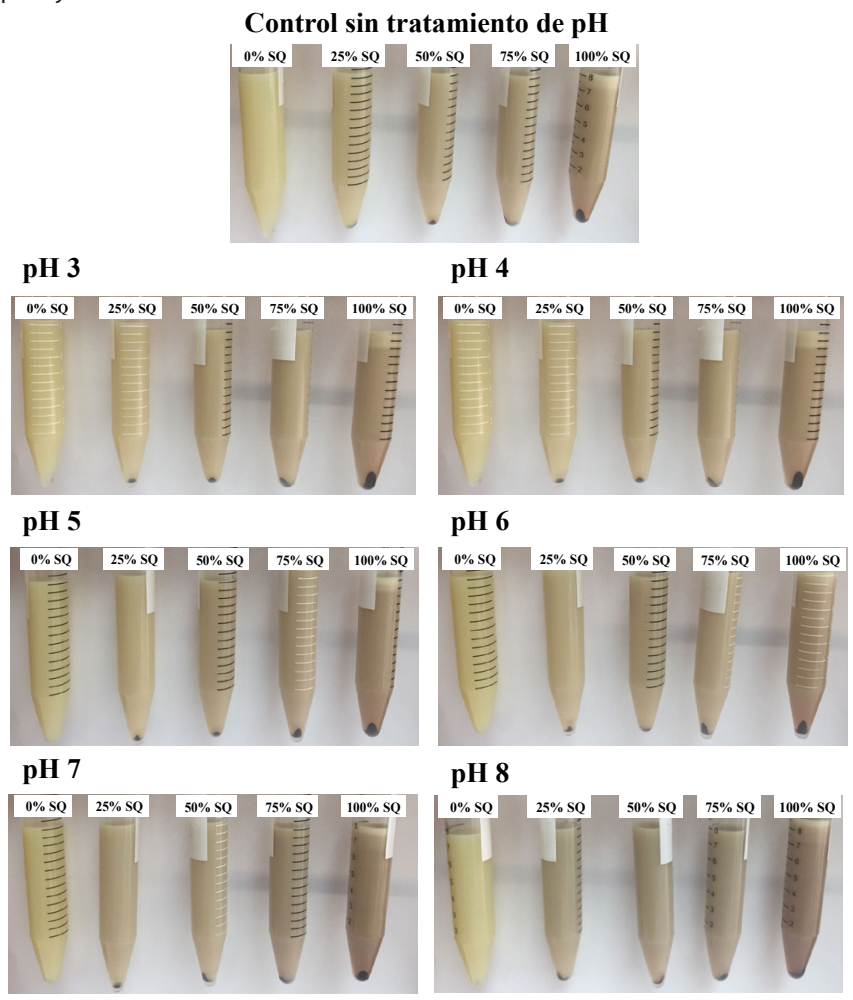

Fuente: Fotografias tomadas por los autores.

\section{Estabilidad oxidativa de las nanoemulsiones}

La estabilidad oxidativa de las nanoemulsiones se determinó mediante la prueba de las sustancias reactivas al ácido tiobarbitúrico (TBARs) bajo condiciones aceleradas de almacenamiento $\left(50^{\circ} \mathrm{C}\right)$, tal como se observa en la figura 5 . Al día cero de almacenamiento, todas las nanoemulsiones presentaron la misma concentración de TBARs independiente del porcentaje de saponinas de quillay (0-100\% SQ); sin embargo, al día 15 de almacenamiento se observó que la adición de saponinas de quillay (25-100\% SQ) retardó la formación TBARs en relación con la nanoemulsión control (0\% SQ) que no contenía saponinas. Al día 30 de almacenamiento todas las nanoemulsiones presentaron un aumento de la concentración de TBARs observándose valores similares entre ellas (figura 5). Si bien es sabido que el uso de surfactantes aniónicos en la elaboración de nanoemulsiones puede aumentar su oxidación lipídica debido a que atraen electroestáticamente a los iones metálicos de transición (McClements y Decker, 2018), el estudio de Uluata et al. (2015) 
FIGURA 5. Concentración de sustancias reactivas al ácido tiobarbitúrico (TBARs) de las nanoemulsiones con diferentes porcentajes de saponinas de quillay (SQ) $(0-100 \% \mathrm{p} / \mathrm{p})$ durante el almacenamiento a $50^{\circ} \mathrm{C}$. Los valores se expresan como promedio y diferencias significativas.

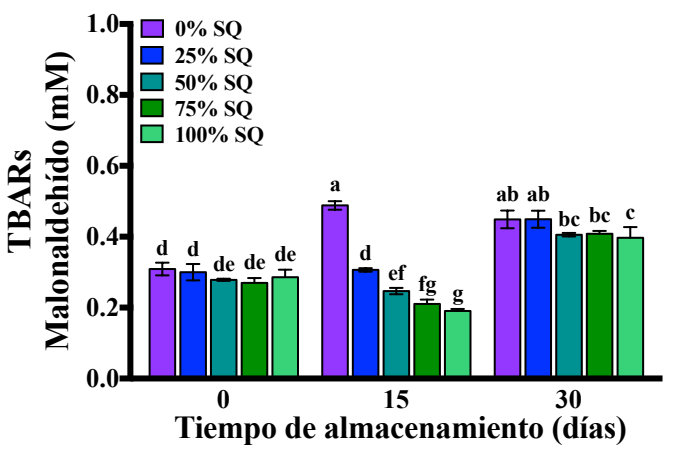

Fuente: Elaboración de los autores.

demostró que las nanoemulsiones estabilizadas con saponinas (surfactante aniónico) muestran una mejor estabilidad frente a la oxidación de los lípidos en relación con aquellas estabilizadas con surfactantes sintéticos (Tween 80 - no iónico), lo cual atribuyeron a su capacidad para frenar la acción de los radicales libres. Lo cual también se pudo observar en nuestro estudio durante los primeros días de almacenamiento en condiciones aceleradas. Por lo tanto, las saponinas de quillay podrían retardar el inicio de la oxidación lipídica lo que representa una ventaja tecnológica frente al surfactante sintético.

\section{Conclusiones}

El remplazo de un surfactante sintético (Tween 80 ) por otro natural (saponinas de quillay) afecta las propiedades físicas de las nanoemulsiones. El uso de saponinas de quillay es menos eficiente en la obtención de nanoemulsiones con tamaños de partícula $<100 \mathrm{~nm}$, sin embargo, tiene un efecto menor sobre el índice de polidispersidad. La mezcla Tween 80-saponinas de quillay modifica los valores de potencial zeta de las nanoemulsiones debido a la competitividad entre los distintos surfactantes por su adsorción en la interfase aceite-agua. Todas las nanoemulsiones presentan una buena estabilidad física durante su almacenamiento al no observarse indicios de formación de cremado durante 30 días. La variación del pH (3-8) no modifica el tamaño de partícula de las nanoemulsiones ni la formación de crema luego de un proceso de centrifugación. Por otro lado, el remplazo por saponinas de quillay mejora la protección frente a la oxidación lipídica durante el almacenamiento en condiciones aceleradas en comparación con el uso del surfactante sintético (Tween 80). Por lo tanto, se puede concluir que las saponinas de quillay son un surfactante natural eficaz que puede ser utilizado en la elaboración de nanoemulsiones estables físicamente y frente la oxidación lipídica lo cual permite su incorporación en una gran variedad de alimentos. 


\section{Referencias}

Arancibia, C., Riquelme, N., Zúñiga, R., Matiacevich, S. (2017). Comparing the effectiveness of natural and synthetic emulsifiers on oxidative and physical stability of avocado oil-based nanoemulsions. Innovative Food Science \& Emerging Technologies, 44(1): 159-166. http://dx.doi.org/10.1016/j.ifset.2017.06.009

Bai, L., Huan, S., Gu, J., McClements, D. J. (2016). Fabrication of oil-in-water nanoemulsions by dual-channel microfluidization using natural emulsifier: saponins, phospholipids, proteins, and polysaccharides. Food Hydrocolloids, 61(1): 703-711. http://dx.doi.org/10.1016/j.foodhyd.2016.06.035

Bortnowska, G., Balejko, J., Tokarczyk, G., Romanowska-Osuch, A., Krzemińska, N. (2014). Effects of pregelatinized waxy maize starch on the physicochemical properties and stability of model low-fat oil-in-water food emulsions. Food Hydrocolloids, 36(1): 229-237. http://dx.doi.org/10.1016/j.foodhyd.2013.09.012

Böttcher, S., Drusch, S. (2016). Interfacial properties of saponin extracts and their impact on foam characteristics. Food Biophysics, 11(1): 91-100. http://dx.doi.org/10.1007/s11483-015-9420-5

Chung, C., Sher, A., Rousset, P., Decker, E. A., McClements, D. J. (2017). Formulation of food emulsions using natural emulsifiers: utilization of quillaja saponin and soy lecithin to fabricate liquid coffee whiteners. Journal of Food Engineering, 209(1): 1-11. http://dx.doi.org/10.1016/j.jfoodeng.2017.04.011

Gupta, A., Burak-Eral, H., Alan-Hatton, T., Doyle, P. S. (2016). Nanoemulsions: formation, properties and applications. Soft Matter, 12(1): 2826-2841. http://dx.doi.org/10.1039/C5SM02958A

Luo, X., Zhou, Y., Bai, L., Liu, F., Zhang, R., Zhang, Z., Zheng, B., Deng, Y., McClements, D. J. (2017). Production of highly concentrated oil-in-water emulsions using dual-channel microfluidization: use of individual and mixed natural emulsifiers (saponin and lecithin). Food Research International, 96(1): 103-112. http://dx.doi.org/10.1016/j.foodres.2017.03.013

McClements, D. J. (2015). Food emulsions: Principles, practices, and techniques, 3a ed. CRC press and Taylor \& Francis Group.

McClements, D. J., Gumus, C. E. (2016). Natural emulsifiers: biosurfactants, phospholipids, biopolymers, and colloidal particles: Molecular and physicochemical basis of functional performance. Advances in Colloid and interface Science, 234(1): 3-26. http://dx.doi.org/10.1016/j.cis.2016.03.002

McClements, D. J., Jafari, S. M. (2017). Improving emulsion formation, stability and performance using mixed emulsifiers: A review. Advances in Colloid and Interface Science, 251(1): 55-79. http://dx.doi.org/10.1016/j.cis.2017.12.001

McClements, D. J., Bai, L., Chung, C. (2017). Recent advances in the utilization of natural emulsifiers to form and stabilize emulsions. Annual Review of Food Science and Technology, 8(1): 205-236.

http://dx.doi.org/10.1146/annurev-food-030216-030154

McClements, D. J., Decker, E. (2018). Interfacial antioxidants: A review of natural and synthetic emulsifiers and coemulsifiers that can inhibit lipid oxidation. 
Journal of Agricultural and Food Chemistry, 66(1): 20-35.

http://dx.doi.org/10.1021/acs.jafc.7b05066

Montes de Oca-Ávalos, J. M., Candal, R. J., Herrera, M. L. (2017). Nanoemulsions: stability and physical properties. Current Opinion in Food Science, 16(1): 1-6. http://dx.doi.org/10.1016/j.cofs.2017.06.003

Öztürk, B., Argin, S., Ozilgen, M., McClements, D. J. (2014). Formation and stabilization of nanoemulsions-based vitamin E delivery systems using natural surfactants: quillaja saponin and lecithin. Journal of Food Engineering, 142(1): 57-63. http://dx.doi.org/10.1016/j.jfoodeng.2014.06.015htt

Öztürk, B., McClements, D. J. (2016). Progress in natural emulsifiers for utilization in food emulsions. Current Opinion in Food Science, 7(1): 1-6.

http://dx.doi.org/10.1016/j.cofs.2015.07.008

Raikos, V., Duthie, G., Ranawana, V. (2016). Comparing the efficiency of different food-grade emulsifiers to form and stabilize orange oil-in-water beverage emulsions: influence of emulsifier concentration and storage time. International Journal of Food Science and Technology, 52(2): 348-358.

http://dx.doi.org/10.1111/ijfs.13286

Salvia-Trujillo, L., Soliva-Fortuny, R., Rojas-Graü, M. A., McClements, D. J., MartínBelloso, O. (2017). Edible nanoemulsions as carriers of active ingredients: A review. Annual Review of Food Science and Technology, 8(1): 439-466.

http://dx.doi.org/10.1146/annurev-food-030216-025908

Uluata, S., McClements, D. J., Decker, E. (2015). Physical stability, autooxidation, and photosensitized oxidation of $\omega-3$ oils in nanoemulsions prepared with natural and synthetic surfactants. Journal of Agricultural and Food Chemistry, 63(42): 9333-0340. http://dx.doi.org/10.1021/acs.jafc.5b03572

Uzoigwe, C., Burgess, J. G., Ennis, C. J., Rahman, P. K. (2015). Bioemulsifier are not biosurfactants and require different screening approaches. Frontiers in Microbiology, 6(1): 245-251. http://dx.doi.org/10.3389/fmicb.2015.00245

WHO-World Health Organization (2006). WHO Food additives series: 56; Safety evaluation of certain food additives. World Health Organization, Ginebra Suiza.

Yang, Y., Leser, M. E., Sher, A. A., McClements, D. J. (2013). Formation and stability of emulsions using a natural small molecule surfactant: Quillaja saponins (QNaturale ${ }^{\circledR}$ ). Food Hydrocolloids, 30(2): 589-596. http://dx.doi.org/10.1016/j.foodhyd.2012.08.008

Zhang, J., Bing, L., Reineccius, G. A. (2015). Formation, optical properties and stability of orange oil nanoemulsions stabilized by Quillaja saponins. LWT-Food Science and Technology, 64(2): 1063-1070. http://dx.doi.org/10.1016/j.lwt.2015.07.034

Zhu, Z., Wen, Y., Yi, J., Cao, Y., Liu, F., McClements, D. J. (2019). Comparison of natural and synthetic surfactant at forming and stabilizing nanoemulsions: tea saponin, quillaja saponin and tween 80. Journal of Colloid and Interface Science, 536(1): 80-87. http://dx.doi.org/10.1016/j.jcis.2018.10.024 